\title{
Belphégor
}

\section{« Nettoyage par le bide ». La Série Noire à l'épreuve des archives}

\section{Adrien Frenay et Lucia Quaquarelli}

\section{(2) OpenEdition}

1 Journals

\section{Édition électronique}

URL : https://journals.openedition.org/belphegor/4342

DOI : $10.4000 /$ belphegor.4342

ISSN : 1499-7185

Éditeur

LPCM

\section{Référence électronique}

Adrien Frenay et Lucia Quaquarelli, « « Nettoyage par le bide ». La Série Noire à l'épreuve des archives », Belphégor [En ligne], 19-2 | 2021, mis en ligne le 05 janvier 2022, consulté le 20 septembre 2022. URL : http://journals.openedition.org/belphegor/4342; DOI : https://doi.org/10.4000/belphegor. 4342

Ce document a été généré automatiquement le 20 septembre 2022.

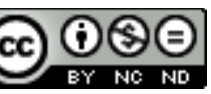

Creative Commons - Attribution - Pas d'Utilisation Commerciale - Pas de Modification 4.0 International - CC BY-NC-ND 4.0

https://creativecommons.org/licenses/by-nc-nd/4.0/ 


\title{
« Nettoyage par le bide ». La Série Noire à l'épreuve des archives
}

\author{
Adrien Frenay et Lucia Quaquarelli
}

Accrochage avec mon éditeur. "Coupe, taille et rogne, il me dit, sinon tes lecteurs vont se faire vieux. "

A mon tour d'en baver. Ça n'est que justice. Mais comment expédier en trois lignes Jim Phelan?

Marcel Duhamel, Raconte pas ta vie, 1972

1 En 1950, pour gagner un abonnement de trois mois à Paris-Match, on pouvait jouer au «Jeu de la Série Noire » : inventer un titre de roman policier « du type Série Noire » et l'envoyer à la rédaction. Parmi les meilleurs titres de la semaine du 21 octobre nous trouvons par exemple «Ça sent le cadavre» ou «Gare à tes pieds, mignonne " ou encore « Nettoyage par le bide ». Si, en 1950, on peut s'amuser à imaginer des titres de romans Série Noire, c'est surement parce que les romans de la collection créée par Marcel Duhamel en 1945 bénéficient d'une identité générico-sérielle suffisamment établie et ancrée dans le lectorat pour qu'on puisse en poursuivre le mouvement (et le "jeu » sériel) en dehors des bureaux de Gallimard ; mais c'est sans doute aussi parce que tout lien entre le titre du roman français et son "original » (américain) est perçu comme accessoire, inintéressant ou non pertinent. Autrement dit, les titres des romans Série Noire sont un des éléments sinon fondateurs du moins révélateurs d'une construction éditoriale et narrative toute française du roman noir. Ils s'insèrent dans un ensemble plus large de pratiques éditoriales qui garantissent ce que J.-M. Gouanvic appelle un " reclassement ${ }^{1}$, du roman hard boiled au roman noir, qui n'est pas seulement l'effet d'une réhistoricisation ou d'une recontextualisation culturelle, sociale ou encore littéraire : il est, surtout, un processus de "mise en série » accompli par le biais d'une sélection et d'une réorganisation d'un certain nombre de traits narratifs dominants et singularisants issus d'une matière américaine hétérogène ${ }^{2}$ qui dépasse le strict cadre du hard-boiled. Il s'agit même parfois, comme nous l'avons montré ailleurs ${ }^{3}$, d'une pratique de sérialisation qui peut s'affranchir de l'existence d'un original américain : on peut faire américain par simple "effet de traduction", par simulation traductive, par authenticité exhibée et présumée. 
2 Or, poursuivre les traces de la machine éditoriale qui garantit la création et l'affirmation de l'identité générico-sérielle de la Série Noire, en lire les indices pour en observer les engrenages et les mettre «en mouvement ${ }^{4}$ est une expérience dépaysante à plusieurs égards.

3 D'abord parce que les archives consultées sont lacunaires, sommairement classées et relativement peu étudiées - comme c'est souvent le cas pour les productions culturelles populaires -, ce qui ne nous a pas permis d'éclairer toutes les étapes du travail éditorial. Ensuite, parce qu'on y découvre un paysage inattendu, dans lequel le geste traductif joue un rôle performatif et créatif constitutif et, aussi, parce que ce geste se fait de manière collective, au-delà de tout dimension dérivative, secondaire et subordonnée et, parfois, de manière fictive ${ }^{5}$. La traduction apparait en fait être non seulement une des modalités de circulation des textes et des imaginaires, capable de modifier le champ littéraire d'« accueil », mais aussi, et surtout, une pratique d'écriture collective qui en permet leur production sur place; pratique qui brouille les notions, largement acquises et répandues dans la théorie de la traduction, d' " original » et de « texte source » - tout comme celles, contigües, de fidélité, respect, équivalence... Ainsi, si c'est la nature seconde de la traduction qui « fait l'original $»^{6}$, qui le fige une fois pour toute dans son unicité et son autorité en se plaçant en aval de son mouvement créatif et en permettant un parcours "à rebours » (on traduit après et d'après l'original), alors tout acte traductif qui corrompt le protocole dérivatif, s'insérant dans la matière narrative avec la force et la liberté inventive de la création, corrompt l'original (sa nature et sa souveraineté) et modifie partant les équilibres de pouvoir entre auteur et traducteur. De plus, si l'atelier du traducteur s'avère être habité et animé par plusieurs figures éditoriales, dont les fonctions se chevauchent sans cesse, c'est tout le processus de création qui doit être repensé et reconstruit.

4 Utiliser les archives de la Série Noire pour «remonter aux origines ${ }^{7}$ du geste d'invention et de création des romans se révèle ainsi être une aventure bizarre, au fil de laquelle auteur et traducteur non seulement intervertissent leurs positions, leurs statuts et leurs fonctions, mais finissent par disparaitre, à la faveur d'un travail collaboratif où la fonction-auteur semble être davantage exercée par l'équipe éditoriale toute entière; au cours de laquelle le geste " originaire " se détache progressivement d'une volonté auctoriale conçue comme émanant d'un individu, qu'il soit auteur ou traducteur. L'acte créatif n'est plus l'affaire d'une seule personne et la traduction (collective, créatrice, "première ») devient le creuset où s'élabore le roman noir « français ».

\section{L'affirmation d'un modèle sériel}

5 L'effet de collection - c'est-à-dire la possibilité offerte au lecteur de reconnaître un texte comme faisant partie d'une famille textuelle plus large - impose une forte homogénéité (stylistique, générique, thématique) des œuvres. Son efficacité repose «sur la convergence d'effets de transtextualité invitant à lire l'œuvre dans la perspective d'un ensemble cohérent, souvent distribué entre un architexte aux contours vagues et une série d'intertextes ayant valeur de relais prototypique " ${ }^{8}$. C'est par le biais d'un jeu subtil entre répétition et variation, entre reproduction d'un modèle architextuel et sa circulation singularisante que les textes s'insèrent dans un ensemble 
narratif sériel plus vaste auquel ils se référent et qu'ils contribuent à consolider, tissant, au fil des publications, un lien et un pacte spécifiques avec le lectorat :

La relation sérielle oscille toujours entre une dynamique de répétition et un souci de la différence. Chaque récit de genre raconte une autre histoire tout en conservant les mêmes propriétés génériques, chaque œuvre d'un ensemble de récits situés dans un univers de fiction récurrent propose sa propre extension de l'univers et l'enrichit d'autant'.

Or, si la « mise en série » se fait, avant tout, par le choix des textes, c'est-à-dire à partir de modèles, modes et modalités littéraires reconnues comme suffisamment caractérisantes pour faire système (ou comme suffisamment conformes dans leur différence pour l'enrichir), elle demande aussi, souvent, une opération d'adaptation et d'appropriation qui passe par un travail éditorial sur et autour du texte qui change suivant l'évolution de l'affirmation de l'identité sérielle, le paysage éditorial dans lequel elle s'insère et ses éventuelles transformations.

7 Si en 1950 on peut s'amuser avec les titres de la Série Noire, c'est parce que la première étape est franchie : l'identité sérielle est affirmée et installée dans l'horizon d'attente du lectorat. La Série Noire est devenue une « marque » qui évoque un imaginaire stable et reconnaissable de thèmes, de styles, d'intrigues, de valeurs et de titres, qu'on peut s'amuser à reproduire, sans risque de se tromper.

8 C'est d'ailleurs à partir de 1948 que la Série Noire, du statut d'initiative presque individuelle avec deux romans publiés par an, se transforme en entreprise éditoriale chargée de produire «à la maison ${{ }^{10}}^{10}$ une importante quantité de romans : la collection est placée sous l'égide des éditions Gallimard et des éditions Hachette ; Marcel Duhamel obtient un contrat ${ }^{11}$; le rythme de parution et les tirages sont revus à la hausse (jusqu'à 46 parutions annuelles et 30000 exemplaires pour chaque volume); la distribution se diversifie (librairies, gares, kiosques) ; le format, la pagination et le prix se stabilisent (couverture cartonnée avec jaquette ; 256 pages ; 135 francs).

9 En somme, à partir de 1948, Marcel Duhamel se trouve à la tête d'une entreprise qui bénéficie rapidement d'une "image de marque » formalisée et lisible, d'une position forte dans le marché éditorial et d'un processus de production établi. Le travail y est mené grâce à l'activité, en plus des quelque 80 traducteurs dits extérieurs, d'une équipe interne ${ }^{12}$ de plus en plus importante parfois appelée «brain trust $»^{13}$ : Minnie Danzas, Janine Hérisson, Jeannie Chauveau et Odile Lagay, bientôt rejoints par d'autres collaborateurs comme René Lelu à partir de $1953^{14}$.

10 Sept ans plus tard, en septembre 1955, la Série Noire fête ses dix ans (et 272 volumes publiés). Marcel Duhamel décide de publier un premier catalogue de la collection pour, écrit-il dans l'introduction, « aider » libraires et lecteurs "à s'y reconnaitre », pour les aider à se retrouver dans une "multitude de titres, de scénarios, de noms d'auteurs et de personnages $»^{15}$. Pour les aider, les guider en quelque sorte, à tisser les fils d'une construction sérielle, désormais accomplie, à partir d'un cadre identitaire fort: il choisit un domaine narratif de référence (la littérature d'évasion), un hypotexte suffisamment provocateur pour dire l'originalité de la série (Le Catalogue des Armes et des Cycles de Saint-Etienne), un public cible (les intellectuels et les professions libérales) et égrène une liste rassurante d'indices de respectabilité et de légitimation - des lecteurs d'autorité (Gide, Colette), des acolytes de renom (Pierre Mac Orlan, Marcel Achard, Pierre Lazareff, Professeur Mondor), des appréciations enthousiastes provenant du monde universitaire international. 
11 La Série Noire est certes, telle son hypotexte fictif, une extraordinaire boîte à " ustensiles à trucider »: un univers d'évasion criminelle, qui nourrit les rêves de ses lecteurs et leur soif d'aventure; une scène dangereuse où évoluent des bagarreurs, «pas forcement tout bons ou tout mauvais ». Mais elle est aussi en 1955, nous dit Duhamel dans l'introduction, un ensemble de "documents vivants", capables de témoigner d'une époque et d'un monde et, ainsi, amenés à se transformer. Marcel Duhamel fait ainsi état de l'existence d'un modèle sériel si installé, cohérent et identifiable qu'il est possible d'en parcourir rétrospectivement l'affirmation et le succès, d'en dessiner les contours, mais aussi d'en annoncer la transformation et le renouvellement, car le « principal souci » de l'équipe de la Série Noire en 1955 est bien celui d'introduire dans la collection la " couleur locale »: "Choisir parmi les quelque cent manuscrits tant français qu'américains qui nous parviennent tous les mois, les plus authentiques ${ }^{16}$.

12 En 1950, l'identité « américaine » de la collection n'est plus à prouver ou à justifier et on peut s'amuser à inventer des titres de romans noirs « Série Noire » en faisant du faux américain en français avec facilité et satisfaction ludique ; en 1955, on peut faire du véritable noir français, sans que cela mette en discussion ni l'authenticité de la narration ni celle de la collection. Pour le dire autrement : en 1955 on peut chercher et trouver l'authenticité «noire » tant dans les romans américains que dans les romans français. C'est d'ailleurs la tâche principale de la Série Noire : faire authentique en y ajoutant la couleur locale. Faire authentique sans original américain; faire du roman noir authentique, localement.

La notion d'authenticité est d'ailleurs l'une des plus résistantes et des plus difficiles à saisir dans l'élaboration du modèle du roman noir. Elle revient de manière quasi obsessionnelle dans la correspondance de Marcel Duhamel et dans ses entretiens, présentant toujours une dimension narrative essentielle: elle intervient dans la justification et la légitimation de la présence d'auteurs anglais au tout début de la série par compensation littéraire (humour et violence à l'américaine $e^{17}$ ) ; elle représente la qualité romanesque majeure dont les romans de la série doivent disposer ${ }^{18}$ et même, parfois, le critère générico-sériel décisif pour justifier le refus d'un manuscrit: "Pas suffisamment authentique pour la Série Noire ${ }^{19}$. Rarement l'authenticité est évoquée, par Marcel Duhamel, dans un contexte et avec un souci de relation (représentationnelle ou non) avec une réalité donnée, un espace-temps défini ou une société précise. Rarement il est question d'adhésion à une quelconque réalité américaine. Pour faire un roman noir américain, pour faire américain, il n'est pas toujours nécessaire d'avoir une connaissance directe d'un pays, d'un peuple et d'une société. L'américanité authentique que la Série Noire expose, défend et propose au public français semble être pour l'essentiel une construction française réalisée, dans les bureaux de la Série Noire, à partir d'un travail éditorial à contrainte sérielle et d'un imaginaire européen partagé ${ }^{20}$.

14 Afin de produire de la vraisemblance et de l'authenticité, le cadre rigide de la série pousse les auteurs (américains, anglais ou français, peu importe finalement) à travailler non pas tant sur la base d'une réalité référentielle et/ou expérientielle spécifique - un monde qui existe et dont ils auraient une connaissance directe -, mais à partir des autres textes de la série, qui construisent progressivement ce monde de l'intérieur. Cependant, une condition semble nécessaire à la réalisation du projet sériel dans la première phase d'affirmation du roman noir et au moins jusqu'en $1951^{21}$ : la dimension traductive. Les romans de la Série Noire sont authentiquement américains aussi par 
«effet de traduction»: ils doivent être le résultat (réel ou fictif) d'un travail de traduction. Ils doivent mettre en scène le simulacre d'un original pour garantir et légitimer leur américanité ; ils sont américains parce qu'ils sont traduits de l'américain.

Ce qui change par contre radicalement à partir de 1951, et qui est scellé par l'introduction de Marcel Duhamel au catalogue de 1955, c'est l'entrée dans la collection de romanciers français qui n'ont plus besoin de se cacher derrière un pseudonyme américanisant; l'entrée dans la collection de romans qui ne montrent plus en couverture l'indication «traduit de l'américain». Il s'agit là, avant tout, d'un changement autorisé et garanti par la stabilité du modèle sériel et par la transformation progressive du paysage éditorial français des récits criminels, mais il s'agit aussi de la réaction à une contrainte juridique: la Chambre de Commerce International menace d'envoyer les faux romans américains au Tribunal de commerce pour concurrence déloyale ${ }^{22}$. Les écrivains français n'ont plus la possibilité de recourir à cette fiction traductive mais ils peuvent cependant entrer à la Série Noire puisque leur légitimité et l'authenticité de leurs romans, de tout roman noir au fond, ne peut plus être mise en cause : elle est avant tout une affaire de "couleur", une affaire générico-narrative de sérialisation. En 1955 la logique éditoriale de la mise en série a produit une identité de marque si forte qu'elle en permet la transgression, le renouvellement et l'élargissement; qu'elle permet son ouverture explicite et revendiquée au roman noir français.

Nous avons décidé de suivre les traces de la construction et de l'affirmation de cette identité sérielle à partir des archives, depuis sa fondation en 1945 et jusqu'à la publication du premier catalogue de 1955 ; d'en remonter les sentiers productifs et d'en observer les mouvements et les stratégies depuis l'annonce d'un "genre nouveau » de roman policier, provenant des maitres anglais et américain - publiée en tête de Cet homme est dangereux de Cheyney (2, juillet 1948) ${ }^{23}$-, à travers la stabilisation d'un « idéal» de roman noir américain construit sur la triade action-angoisse-violence tel qu'il est énoncé dans le presque-manifeste de $1948^{24}$, et jusqu'à l'affirmation du roman noir français qui marque l'entrée progressive des écrivains français dans la collection, entre 1951 et 1955.

\section{Une fabrique traductive}

\section{Le fonds}

Nous avons travaillé sur le "Fonds Duhamel/Gallimard ", conservé à la Bibliothèque des Littératures Policières depuis 1984. Il se compose de trois ensembles de documents : des exemplaires de romans en langue étrangère (quasi exclusivement en anglais ou en américain) de 1945 au début des années 1990; une partie de la correspondance de Marcel Duhamel et des notes de services de la collection, principalement pour la période 1945-1966; des manuscrits et feuillets divers qui concernent la période 1955-1992.

18 Il est possible de classer les exemplaires présents ainsi : a) romans traduits et publiés dans la collection; b) romans traduits et publiés dans d'autres collections liées à la Série Noire ou à Marcel Duhamel (comme la "Série Blême", "Carré Noir", «Panique », ou encore « Oscar », chez Denoël) ; c) romans finalement non traduits. Les exemplaires correspondant à la première catégorie sont principalement des hardbacks, 
des paperbacks et des paperback originals. Jusqu'à 1955, ils proviennent majoritairement des États-Unis (202 romans, 74\% du total des romans publiés) et d'Angleterre (41 romans, 15\%). Les 29 romans restants (11\%) sont publiés en français (dont 4 sous pseudonyme américanisant).

L'intérêt principal des exemplaires anglo-américains du fonds est de nous permettre d'observer les traces matérielles laissées sur les volumes à l'occasion du travail d'édition et de traduction afin de suivre de près les étapes par lesquelles passe un roman anglo-américain, depuis sa réception dans les bureaux de la collection jusqu’à sa parution.

Pour les années 1945-1955, le fonds Duhamel permet d'étudier le traitement de 117 romans en langue anglaise ou américaine ${ }^{25}$, répartis sur la quasi-totalité de la période.

Tableau 1. Exemplaires présentant des marques éditoriales préparatoires à la publication dans la collection.

Tableau 1. Exemplaires présentant des marques éditoriales préparatoires à la publication dans la collection

\begin{tabular}{|c|c|c|c|c|c|c|}
\hline Années & $\begin{array}{l}\text { Romans } \\
\text { parus }\end{array}$ & $\begin{array}{l}\text { Romans } \\
\text { traduits }\end{array}$ & $\begin{array}{l}\text { Romans écrits } \\
\text { en français }\end{array}$ & $\begin{array}{l}\text { Romans présents } \\
\text { dans le fonds }\end{array}$ & $\begin{array}{l}\text { Exemplaires } \\
\text { vierges }\end{array}$ & $\begin{array}{l}\text { Romans présentant des } \\
\text { marques }\end{array}$ \\
\hline 1945 & 2 & 2 & 0 & 0 & s/o & $\mathrm{s} / \mathrm{o}$ \\
\hline 1946 & 2 & 2 & 0 & 1 & 0 & 1 \\
\hline 1947 & 2 & 2 & 0 & 0 & $\mathrm{~s} / \mathrm{o}$ & $\mathrm{s} / \mathrm{o}$ \\
\hline 1948 & 14 & 13 & 1 & 2 & 2 & 0 \\
\hline 1949 & 26 & 26 & 0 & 5 & 2 & 3 \\
\hline 1950 & 26 & 24 & 2 & 12 & 4 & 8 \\
\hline 1951 & 39 & 37 & 2 & 16 & 4 & 12 \\
\hline 1952 & 36 & 36 & 0 & 25 & 6 & 19 \\
\hline 1953 & 36 & 30 & 6 & 16 & 3 & 13 \\
\hline 1954 & 46 & 38 & 8 & 22 & 11 & 11 \\
\hline 1955 (sept) & 43 & 33 & 10 & 18 & 5 & 13 \\
\hline Total & 272 & 243 & 29 & 117 & 37 & 80 \\
\hline
\end{tabular}

Quatre-vingts exemplaires présentent des marques attribuables au traducteur ou à l'équipe éditoriale. Parmi eux, certains sont recouverts de ratures et de mentions en marge ${ }^{26}$. À l'inverse, 37 exemplaires sont vierges. L'absence de marque ne signifie pas pour autant qu'il n'y a pas eu d'intervention éditoriale, qui a pu se matérialiser sur un autre exemplaire (une version hardback ou paperback) ou sur un autre état du texte (épreuves préalables à l'édition anglo-américaine, manuscrit), perdu ou conservé par le traducteur ou l'un des membres de l'équipe éditoriale ${ }^{27}$. Le fait que $68 \%$ des exemplaires anglo-américains du fonds pour la période soient annotés (soit 32\% des 243 romans traduits publiés jusqu'en septembre 1955) accrédite l'hypothèse du caractère systématique, ou de plus en plus systématique, de l'intervention éditoriale. De surcroît, l'existence de cas qui témoignent d'une intervention éditoriale importante alors même que les exemplaires présents dans le fonds sont vierges laisse à penser que ce caractère systématique serait même sous-évalué par rapport à la réalité du phénomène ${ }^{28}$.

\section{Les marques}

Les traces qui témoignent du processus éditorial de publication sont aussi diverses que peuvent l'être celles qu'on trouve sur des brouillons ou des épreuves (du trait à l'ajout en marge en passant par la biffure). Elles sont réalisées par des personnes différentes d'un exemplaire à l'autre - même s'il semble possible d'identifier des récurrences à certaines périodes -, en une (même manière d'annoter ou de biffer, même stylo ou 
crayon) ou plusieurs campagnes (plusieurs stylos ou crayons, plusieurs couleurs correspondant à plusieurs manières de marquer l'exemplaire). Elles permettent de reconstruire des intentions de traduction, de correction, d'ajout, de suppression et de substitution. Ces marques sont effectuées tant sur le texte lui-même (ratures, encadrement, soulignement) que dans ses marges (traits et accolades, mots, chiffres, signes divers liés au repérage) ainsi qu'au sein des seuils éditoriaux (identification des agents, mentions et instructions, calculs du nombre de signes).

Il est possible à partir de ce relevé d'identifier transversalement trois ensembles de marques correspondant à trois ensembles d'actions effectuées sur le texte : d'une part, les ratures, encadrements, accolades, mentions et calculs qui visent à prévoir la suppression de parties du textes, avec ou sans substitution, parfois jusqu'à plus du quart du total estimé de signes ${ }^{29}$; d'autre part, les marques liées au travail de traduction, qui correspondent à une gamme variée et hétéroclite d'interventions, allant de la correction de coquille au signalement, par soulignement ou inscription de croix, des difficultés de traduction, avec ou sans proposition ${ }^{30}$; pour finir, un ensemble des marques plus largement liées aux pratiques éditoriales et commerciales extratextuelles (prière de retourner, carte des agents, informations concernant les droits cinématographiques, etc.).

Le relevé et la typologie des marques dessinent la mise en place progressive d'une pratique éditoriale très interventionniste qui met à nouveau le texte publié à l'étranger à l'état de manuscrit - version que l'on peut encore corriger et transformer en vue de sa publication -, et qui se poursuit et se stabilise dans les périodes ultérieures. Un tour d'horizon rapide des exemplaires - très raturés - présents dans le fonds, correspondant aux romans de la collection numérotés de 900 à 2000 (1964-1985), permet de faire l'hypothèse que les dix premières années de la collection sont un moment de construction et affirmation d'un processus de travail éditorial à contrainte sérielle qui perdure et se renforce jusqu'à l'arrivée à la direction de Patrick Raynal. D'ailleurs, à partir des années 1960, les marques sont vraisemblablement réalisées par une seule personne, avec le même crayon, de la même manière (encadrement et croix de saint André). Une note de lecture rédigée par Marcel Duhamel du roman The Travelling Executioners (1964) ${ }^{31}$, écrit par Bernard Newman et publié en 1964, montre explicitement l'existence d'une phase de préparation:

Pas mal de suspense, et pourtant c'est assez mal foutu, mal montré et mal écrit.

Seulement voilà = on s'intéresse aux personnages et on ne lache [sic] pas le bouquin.

Il faudrait : [...]

2) couper $1 / 3$ surtout dans ce qui a trait aux méthodes actuelles du C I de Moscou

[...].

3) couper dans l'histoire, courte drailleurs [sic], d'Alexandra [...].

4) bref, revoir en e<n>tier et retailler le mieux possible ».

\section{La préparation}

25 À partir des documents dont nous disposons et des typologies des marques répertoriées, qui relèvent pour la plupart des tâches éditoriales spécifiques (comptage des signes, mentions d'agents, date d'envoi au traducteur etc.), nous pouvons reconstruire un processus de travail par phases distinctes et successives, bien que menées souvent de manière collaborative, dont la première, de "préparation " 
éditoriale à forte contrainte sérielle, serait mise au point par l'équipe éditoriale avant le travail du traducteur.

Par ailleurs, les exemplaires de la période suivante et certains documents corroborent l'hypothèse, faisant référence explicite à cette pratique éditoriale. La note de lecture citée supra de The Travelling Executioners, par exemple, dit clairement que la volonté de l'éditeur de couper des passages du texte, concrétisée par de nombreuses marques portées sur l'exemplaire de la version anglaise, est préalable à l'envoi au traducteur. De même, l'exemplaire de Broken Angel (1957) ${ }^{32}$, écrit par Floyd Mahannah, qui deviendra Un ange est cassé, traduit par Gilberte Sollacaro, présente-t-il, avant la page de titre, la mention manuscrite suivante : « couper 40 pages au début ».

Faire l'hypothèse d'un texte de travail «préparé » par l'éditeur (l'équipe de rédaction) pour le traducteur avec des instructions de coupes et des conseils ou des propositions de raccord et traduction signifie, avant tout, reconnaitre aux marques éditoriales la fonction de protocole opératoire : elles sont là pour dire au traducteur ce qu'il doit traduire et parfois même comment il doit traduire. Mais elles nous disent aussi autre chose: premièrement, comme nous l'avons déjà observé, que l'original est traité comme un manuscrit, qui arrive à la maison d'édition avant publication pour lecture et editing éventuels (on peut le couper, le réécrire, le commenter, etc.) ; deuxièmement, que le traducteur travaille (traduit) à partir d'un texte déjà " second ", déjà " traduit " en quelque sorte par l'éditeur. La traduction n'est en somme pas seulement un travail collectif en plusieurs mouvements, dont le premier serait régi par l'éditeur, mais elle est aussi un travail d'écriture, collective, à contrainte multiple qui se débarrasse de la "sacralité » et de l'autorité de l'original pour le réduire à l'état de brouillon. Dès lors, l'original semble perdre son statut de "source» unique et se transforme bien plus en matière narrative non achevée sur laquelle on peut intervenir, à plusieurs mains ; une première version à reprendre, amender et corriger pour en permettre la mise en série, c'est-à-dire pour en permettre à la fois l'adaptation et l'appropriation.

\section{La nature des coupes}

L'observation de la stratégie des coupes montre que l'impératif matériel n'est qu'une contrainte parmi d'autres. Si les comptages des caractères que l'on trouve en marge des exemplaires du fonds témoignent d'un réel besoin de respecter une foliation fixe et une dimension donnée (celle des présentoirs Hachette), pour garantir la viabilité économique de la collection et en permettre la mise en circulation, on trouve aussi des romans largement coupés dont le comptage établi sur la version en anglais fait état d'une "taille " conforme, compte tenu même de la hausse de caractère d'ordinaire attendue par le passage au français (le foisonnement). Ainsi, au regard des documents dont nous disposons, la pratique des coupes semble n'être qu'en partie le résultat d'une nécessité de réduction matérielle du texte : elle semble répondre aussi à un ensemble d'impératifs d'ordre stylistico-narratif.

Les coupes servent un dessein générico-sériel plus large: elles participent à la réorientation des textes et à leur reclassement; elles s'avèrent même être un des dispositifs majeurs du processus de sérialisation. Et si elles sont établies au cours de la première étape du travail éditorial, dans la phase de préparation, elles gardent leur force prescriptive souvent jusqu'à la fin du processus de production. 
30 À en juger à partir de notre corpus, les instructions de coupes sont, la plupart du temps, respectées, totalement ou partiellement; elles font office de protocole opératoire jusqu'à la fin du processus de travail.

31 Considérées sous l'angle générique, les indications de coupes notées sur les textes de travail présents dans le fonds pourraient être lues à partir de quatre grandes typologies de transformation narrative ${ }^{33}$. Les romans sont coupés premièrement afin de transformer leur rythme narratif. Les informations jugées redondantes et/ou accessoires sont supprimées. Le traitement de The Blank Wall (1947) ${ }^{34}$ d'Elisabeth Sanxay Holding, dont les indications de coupes prévoient une réduction d'un peu moins de 5\% de nombre total de signes en anglais, montre par exemple la volonté, par le recours à de très nombreuses micro-coupes (un mot, une expression, une demi-phrase) à rendre le roman plus fluide en supprimant des détails. Pour produire la version française, intitulée Au pied du mur, sont donc supprimées par exemple des reformulations (p. 16), des détails concernant la tenue de la protagoniste (p. 24), la localisation très précise de deux personnages (p. 28), la méthode de réfrigération des aliments (p. 35), la posture des locuteurs (p. 37 et 42), les actions réalisées pendant les dialogues (p. 48), les phrases qui synthétisent une action ou un dialogue qui vient d'avoir lieu (p. 103).

À ce recours à des coupes courtes et précises, qui invite les traducteurs à simplifier de manière générale l'écriture de l'auteure, s'ajoute cependant une autre pratique qui correspond à un second type de coupes, souvent plus longues : la dépsychologisation. $\mathrm{Si}$ le roman construit un suspense lié à la capacité de la protagoniste à protéger sa famille, et notamment sa fille Bee et son père, qui tue accidentellement le petit ami dangereux de Bee, il est aussi celui d'une femme au foyer américaine pendant la Seconde Guerre mondiale, laissée seule avec ses enfants pendant que son mari est au front et dont les pensées entremêlent des considérations criminelles et des souvenirs familiaux, des réflexions sur la parentalité, sur la peur de la mort, des moments exprimant l'angoisse de laisser ses enfants sans protection et des moments de révélation à soi de sa propre puissance d'action dans le monde. Face à la nécessité de cacher la culpabilité de son père et de sauver sa fille et sa famille du chantage, l'héroïne trouve l'occasion de construire activement son identité et son rôle au sein de sa famille et de la société. Ce sont très souvent ces passages dont les coupes sont programmées. Sans que leur volume puisse permettre d'affirmer que le roman est radicalement transformé, la version française en minore l'un des aspects qui participent pourtant de la construction fine du suspense - et amoindrit bien évidemment la portée sociale et politique de la version américaine.

On retrouve ces abrègements liés à l'argumentation intérieure, à la délibération, au plan d'action non prononcé par le personnage dans la majorité des romans présentant des marques de coupes : le plan d'action écrit à la troisième personne du singulier mais depuis le point de vue de Whit dans Shear the Black Sheep $\left(1943\right.$, p. 25) ${ }^{35}$, s'il répond à la nécessité d'accélérer le rythme du roman en privilégiant l'action, s'apparente aussi à la suppression d'un discours intérieur qui vise à décrire le personnage par la manière dont il programme cette action. De même, l'équilibre de l'incipit de Mr Denning Drives $N_{0 r t h}{ }^{36}$ est transformé en profondeur par le programme de coupes. Tom Denning rêve qu'il est condamné à mort. La version française ne retient du thème du cauchemar que le strict nécessaire: une scène stéréotypée qui permet d'inscrire au cœur du roman l'obsession du personnage pour le crime qu'il a commis et sa peur d'être découvert. La version anglaise, quant à elle, déploie davantage la complexité psychologique du 
meurtrier, exprimée par une attention particulière aux jeux de regards (de la salle, du jury, du juge, du personnage), au discours intérieur, aux paroles qui pourraient lui être directement adressées et enfin aux brouillages de la frontière qui sépare le rêve de la réalité.

Cette attention à l'amoindrissement du psychologique est par ailleurs une constante qu'on retrouve tant dans la correspondance de Marcel Duhamel que dans les publications ultérieures de la collection, de manière assumée et explicite : la deuxième de couverture des épreuves de Double Take (1972) ${ }^{37}$ de David Craig, publié en Angleterre en 1972 et en Série Noire en 1973 comporte la mention suivante : "coupes: ds [sic] gamberges psycho (surtout la dernière partie) facile à faire et y aller carrément ».

Le troisième type de coupes programmées relève d'une volonté d'affaiblir les enjeux politiques présents dans les romans anglo-américains. Au-delà de la suppression des références à la politique intérieure américaine, ce sont les allusions trop fréquentes et trop précises au communisme ou au fascisme qui sont jugées superflues, c'est-à-dire non conformes au modèle sériel. Si les tensions Est-Ouest sont toujours lisibles, elles ne se manifestent plus en Série Noire que sous la forme d'un cadre stéréotypé au sein duquel l'action criminelle, policière ou d'espionnage, se déroule. Le traitement de The Man Inside (1954) ${ }^{38}$ de M.E. Chaber, dont l'action se déroule dans l'Espagne franquiste, est représentatif de cette volonté de simplification de la lecture des enjeux et du contexte politique de l'action: sont coupées les références au contexte politique américain (p. 80 et 89), les allusions au communisme (p. 138 et 167), au phalangisme (p. 154, 165), à Franco (p. 145, 170 et 173).

Dans le roman de Chaber nous pouvons également trouver des exemples du quatrième type d'abrègement, consistant à réaliser une action de domestication et de simplification culturelles. De nombreuses références historiques, sociales et culturelles sont coupées quand elles risquent, en plus de ralentir le rythme de l'action, de n'être pas comprises par le lectorat français. Au lecteur de Voué au blanc-bleu on fait grâce, par exemple, de la description des magazines espagnols (p. 94-95), des considérations linguistiques portant sur des mots espagnols (p. 188-189), des paroles d'une chanson suivie de sa traduction (p. 125-126), d'une plaisanterie faisant référence à Don Quichote (p. 129) ainsi que de toutes les références littéraires ultérieures au Quichotte ou à d'autres auteurs (p. 140-141 et 146-147).

Ces quatre types de coupes, auxquels on procède parfois dans le même roman, forment un instrument puissant de réorientation sérielle : ils renforcent les effets de cohérence entre les différents romans publiés dans la Série Noire et servent un idéal architextuel précis. Le traitement du roman écrit par W.P. McGivern en 1953 intitulé The Big Heat (1953) ${ }^{39}$ en est représentatif. Le protagoniste, policier incorruptible dont la femme est assassinée, cultive un intérêt marqué pour la philosophie (Croce et Kant notamment), s'intéresse aux lectures et à la bibliothèque de la victime (p. 15, 17-18, 21-23, 77, 86, 189); spécule volontiers, cherche à élucider rationnellement (p. 15, 23); décrit précisément les intérieurs (p. 14-16, 24, 87), les paysages qu'il traverse, les itinéraires (p. 19, 31, 37, 48, 93), les hommes et les femmes qu'il rencontre (p. 17, 20, 43), les clients des bars qu'il fréquente (p. 25) ; s'épanche sur ses affaires en cours (p. 30, 34-36), sur la police (p.60), son rôle (p. 31), son organisation (p. 63) et sur la corruption; réfléchit à sa manière rationnelle de gérer ses émotions et notamment sa colère (p. 44) ou choisit volontairement d'abandonner la philosophie pour céder à la violence (p. 77-80, 86); réorganise ses pensées des événements passés pour y voir plus clair $($ p. $45,130,132)$; se 
projette dans l'avenir d'une ville nettoyée de la corruption (p. 57), enfin en général entoure chaque choix et chaque action d'un ensemble de pensées et de description. C'est donc précisément ce qui est coupé dans Coup de torchon, publié aussi en 1953 et traduit par Janine Hérisson. Dans le roman de McGivern, les coupes liées à l'épaisseur du protagoniste, ainsi que celles liées à d'autres personnages (pensées de Larry p. 98 et 109, de Stone p. 101, du Juge McGraw p. 126) ou au contexte historique, social et politique (discussion politique p. 46, expression anticommuniste p. 144, pouvoir de la presse aux États-Unis pp. 60-61) orientent le roman vers le crime et l'action, au détriment de la profondeur psychologique et intellectuelle du protagoniste, dont le basculement du rationnel et du contrôle vers la violence est l'un des intérêts diégétiques en anglais. Les plus de 42000 signes qu'on prévoit de couper, sur un total d'environ 430000, sont consacrés à des motifs, des références et des scènes qui ne correspondent pas à un roman d'action au rythme enlevé dont l'intérêt central n'est plus l'élucidation.

Ce geste de réorientation, qui apparaît dans ce cas comme l'application rigoureuse des marqueurs éditoriaux et commerciaux de la collection tels qu'ils sont évoqués dans les textes rédigés par Marcel Duhamel, est par ailleurs redoublé par l'utilisation de l'argot. En dehors des cas, sans doute pas majoritaires, où l'argot parisien apparaît à l'éditeur et au traducteur comme un équivalent du slang, des mots d'argots sont ajoutés. C'est le cas des traductions françaises en Série Noire des romans de J.H. Chase (ainsi que ceux écrits sous pseudonymes) qui sont pourtant rédigés dans un anglais dénué de ce slang. À propos du traitement de The Maltese Falcon et de Red Harvest, J.-M. Gouanvic conclut que les traducteurs (respectivement H. Robillot; J.-P. Herr et H. Robillot) forcent le trait alors que les textes de Dashiell Hammett sont familiers tout au plus ${ }^{40}$. Comme les coupes, le recours à l'argot permet, à partir d'un matériau hétérogène, d'offrir au lecteur une expérience plus homogène : les deux auteurs anglais qui lancent la vogue du roman américain à la Série Noire, Chase et Cheyney, aussi différents soient-ils en termes de langue et de vision du monde, se rapprochent ne serait-ce qu'un peu dans la collection et partagent du même coup un ensemble de références linguistiques et stylistiques avec les romans bien antérieurs de Dashiell Hammett.

\section{Auctorialité collective}

39 Considérant ces transformations importantes d'ordre narratif, stylistique et linguistique, quel est alors le statut de l'auteur et de son texte? L'équipe de Marcel Duhamel corrige les coquilles sur les exemplaires sélectionnés, propose des traductions de mots ou d'expressions volontiers argotiques, quel que soit le registre de langue ou le sociolecte du roman anglo-américain, donne des instructions de coupe, avec ou sans substitution, avec ou sans raccord. Le texte anglo-américain est par ailleurs librement modifié par les membres d'une équipe éditoriale où les rôles sont partagés. Les fonctions de traduction sont assumées à la fois par le directeur de la collection, par une partie des membres de l'équipe interne ainsi que par le groupe externe de traducteurs. Une même personne peut donc être à l'origine de la proposition d'un roman, rédacteur de la note de lecture d'un autre, traductrice et reviewer d'un troisième, seule ou bien en collaboration avec quelqu'un d'autre - ou encore assumer l'ensemble de ces rôles pour un même roman. Il est difficile dans ces conditions d'attribuer à l'un ou à l'autre la paternité des nombreuses interventions réalisées sur le texte anglo-américain. 
Ce ne sont donc pas les hésitations et les repentirs de l'auteur anglophone auxquels nous confronte le fonds Gallimard/Duhamel, ni même les tentatives successives de traductions, mais le travail de l'éditeur à l'œuvre en tant qu'auteur collectif. Dans le cas de la Série noire, l'œuvre d'un auteur anglo-américain revient à l'état de manuscrit et de brouillon: soumise à nouveau au travail de l'équipe éditoriale (française), elle est préparée par l'éditeur avant même la traduction. Elle devient le brouillon d'une œuvre collective dont l'autorité se conçoit désormais en partage. Avant même de passer par les mains du traducteur, le roman anglo-américain est façonné par une fabrique sérialisante, co-auteure de toutes les œuvres. L'autorité des romans de la série est finalement assumée, que ce soit au niveau du détail des textes que de la portée des œuvres, par l'équipe éditoriale tout entière.

\section{Réviser, Réécrire, Reparler}

Les interventions éditoriales touchent aussi au travail du traducteur: les transformations, modifications et réécritures ont lieu aussi après réception du manuscrit du traducteur. Plusieurs documents laissent en effet à penser que le processus de publication à la Série Noire prévoit, avant mais aussi après le travail du traducteur, des étapes qui visent à la cohérence sérielle, qu'il s'agisse d'impératifs logistiques ou narratifs. Avant d'être transmis au traducteur, le texte anglais ou américain est préparé; après que la traduction a été rendue, celle-ci semble être reprise et davantage travaillée par l'équipe interne.

Dans un rapport intitulé "COMPTABILITÉ: Direction de la Série Noire ", Marcel Duhamel décrit à Claude Gallimard le 16 septembre 1949 le fonctionnement de la collection et aborde la question de la modification des traductions réalisées par les traducteurs. Il y détaille les frais et les bénéfices attendus et développe les aspects liés à la traduction et au secrétariat. Dès la première page, lorsqu'il évoque la question des bénéfices, survient incidemment, pour rehausser l'importance des frais à venir si une nouvelle collection - la "Série Blême » - devait être lancée, la mention d'une étape de publication à la Série Noire qui semble d'importance : la révision : « (À l'heure actuelle, nous avons 25 volumes à réviser complètement et arrivons à peine à maintenir le rythme »). Plus loin dans le même document, quand il s'agit d'aborder la rémunération des traducteurs, la caractérisation de Jacques-Laurent Bost va dans le même sens: "Jacques-Laurent Bost qui n'est pas mauvais, mais demande à être revu entièrement, ne veut plus travailler à moins de 60.000 francs ». Une fois la traduction rendue par le traducteur, une étape de révision semble s'insérer dans le processus de publication, qui est, selon les traducteurs, plus ou moins importante.

Le long « Rapport sur la Série Noire », non daté, mais vraisemblablement écrit dans le courant de l'année $1950^{41}$ revient encore sur ces points. Pour faire état de la faiblesse de sa rémunération comparée au travail que lui et son équipe fournissent, Marcel Duhamel, sur un ton véhément, écrit ceci :

Je répète, jusqu'ici on ferme les yeux sur le travail énorme que représente le choix, parmi des centaines de livres, de 50 à 60 livres par an (avec l'avance d'un an exigée), la traduction des volumes, la nécessité une fois traduits de les réécrire (on saura plus tard pourquoi), de les faire retaper, de revoir les premières et secondes épreuves, de maintenir un contact permanent avec chaque auteur et 40 agents new-yorkais, anglais et français, etc... etc... 

pour les volumes choisis la "nécessité une fois traduits de les réécrire ", mais cette étape de réécriture ressurgit dans les pages suivantes. À la page 2, à l'occasion d'une comparaison entre le travail éditorial de la «Série Blanche » et celui de la Série Noire, Marcel Duhamel assure, parlant des livres, que «c'est plus compliqué pour un des nôtres que pour la Série Blanche car nous les écrivons à la maison ». À la page 4, dans une section intitulée " EN DETAIL », après avoir fait une liste des traducteurs « dignes de ce nom $»^{42}$, Duhamel indique le coût d'une traduction: «Je les paies 50.000 frs. Soit $50 \times 2=$ 100.000 par mois $^{43}$. Le tout entièrement à refaire ou presque par 2 excellentes traductrices que je paie $2 \times 50.000=100.000$ ".

Un dernier ensemble de six pages de la même époque abordant les mêmes questions permet de comprendre clairement le rapport qu'entretient Marcel Duhamel à la matière anglo-américaine qu'il sélectionne pour créer la Série Noire. Dans la section intitulée Addendum, il reproduit à nouveau les calculs cités précédemment, mais utilise un vocabulaire spécifique pour nommer les textes traduits par les traducteurs: «Révision de chaque manuscrit (15 jours de travail en moyenne par manuscrit, quand ce n'est pas un mois). Retapage après correction, la plupart du temps, - soit 10 à 12.000 francs de frais supplémentaires». Le travail effectué par les traducteurs est nommé « manuscrit ». L'emploi n'est pas impropre, mais la quantité de travail que nécessite ce manuscrit pour passer à l'état d'épreuve interroge. S'il faut 15 jours de travail à deux personnes, et parfois un mois, et ce même si cette quantité de travail est exagérée pour le bien de la force persuasive de la demande que le directeur exprime, c'est qu'il ne s'agit pas simplement d'une simple étape de correction ou de relecture. Il s'agit bien ici vraisemblablement, comme Marcel Duhamel le mentionne, de manuscrit de traduction qu'il s'agit de "réviser complètement ", qui sont " entièrement à refaire ", de volumes qui présentent « la nécessité une fois traduits de les réécrire » «à la maison » « par des spécialistes ».

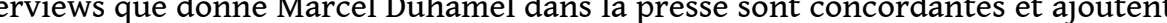
une dimension supplémentaire à cette intervention de l'éditeur : lorsqu'Any Bonneval l'interroge pour la Gazette des Lettres le 5 août 1950 sur la manière qu'à l'équipe de traduire les romans américains, il utilise une image qui fait de son équipe une sorte de troupe de comédiens ou d'acteurs faisant une répétition :

Lorsque le livre nous est réexpédié par les traducteurs de l'extérieur, nous les revoyons nous-mêmes, mot à mot, nous en pesons les termes, les expressions, nous le «reparlons» en quelque sorte. C'est un travail passionnant. Nous prenons parfois, chacun à son compte, un personnage. Vous comprenez, c'est le langage parlé qui est important dans ces livres, c'est la vie de tous les jours, la vraie vie, non conformiste, vivante.

L'éditeur dit réviser, réécrire, écrire et même reparler les romans qu'il publie, à partir de ce qui n'est plus qu'une version de travail, une sorte de scénario ou de texte théâtral que le réalisateur ou le metteur en scène respecte ou non, modifie, amende et transforme à son gré - comme une partition, transcription d'une œuvre que seule l'exécution permet de faire advenir. Co-auteurs des romans traduits dans la collection, les membres de l'équipe de Marcel Duhamel se font aussi les interprètes de romans « reparlés » en scénarios, devenus presque, malgré leurs différences, des épisodes d'une série, à tous les sens du terme, entremêlant des fils hétérogènes sous forme d'une suite parfois feuilletonnante de produits commerciaux calibrés, caractérisée par le retour d'éléments dont la récurrence est rehaussée par les coupes et l'argot. 


\section{Conclusion} transforme les produits semi-traités ou les matières premières en objets manufacturés destinés à être livrés au commerce ${ }^{44}$. Elle devient une fabrique, qui reçoit de l'étranger des «produits semi-traités », c'est-à-dire déjà édités au Royaume-Uni ou aux États-Unis, pour les transformer, à l'aide d'une stratégie traductive précise servant un modèle générico-sériel spécifique. Ou encore (parfois et progressivement), elle est le lieu même où l'artefact sériel, le roman noir, se produit, à partir d'une matière première toute française, d'abord cachée (faux auteurs américains, fausses traductions) puis révélée et présentée comme « authentique ». En effet, si avant 1951 la traduction joue un rôle majeur dans la construction de la Série Noire, à la fois comme processus de traitement des textes et comme élément de sa construction narrative - en en attestant, par simple présence (réelle ou fictive), l'authenticité -, après la publication du roman d'Yvan Dailly ${ }^{45}$, sa fonction de témoin irréfutable d'américanité, sa responsabilité de "garant » de l'effet de série, s'avère moins nécessaire, puisque l'identité sérielle est suffisamment établie, stable et reconnue pour se justifier et s'autoriser d'elle-même. À partir de 1951, en somme, plus besoin de faire semblant, plus besoin de faire américain: l'authenticité des romans n'est pas à démontrer; elle n'est plus qu'une affaire de « couleur».

51 Pourtant, la traduction, pratique d'écriture collective à forte dominante créative, ne cesse jamais d'être le creuset où s'élabore le roman noir. Elle n'est jamais simple production dérivée - établie après et d'après l'original - : elle est geste premier, mouvement de création et outil essentiel de sérialisation et de reclassement générique. Elle constitue la condition même de possibilité de la Série Noire, car elle permet la réorientation (narrative, stylistique, générique, idéologique) nécessaire pour faire un roman Série Noire. Et elle ne se réalise pas dans l'atelier d'un traducteur solitaire et studieux, mais au sein d'une entreprise éditoriale qui en décide les stratégies et les contraintes, remet le texte anglophone à l'état de brouillon et se donne des instructions 
(injonctions) de travail précises : ton, argot, coupes, rythme serré, pas de psychologie, pas de politique, pas de dépaysement culturel.

En Série Noire, l'œuvre étrangère passe à travers plusieurs "brouillons postérieurs ", pour reprendre l'expression de Tiphaine Samoyault ${ }^{46}$, fruits d'une pratique traductive pilotée et contrainte qui se fait par le biais de différentes campagnes de préparation, écriture, réécriture, menées par une diversité grandissante d'acteurs-auteurs, qu'il n'est toujours pas facile d'identifier. Qu'il n'est même probablement pas utile ni pertinent de saisir avec précision. Car ce qui se produit dans ce passage de l'atelier du traducteur à la fabrique éditoriale et sérielle qu'est la Série Noire est la mise en échec, par saturation, tant de l'unicité et de l'originalité de l'œuvre que du caractère sacré et créateur de l'auteur et de celui (tout récent) du traducteur.

Ce qui est en jeu n'est pas seulement le "pluriel de l'œuvre " comme il existe dans toute relation d'interprétation (et la traduction en est une ${ }^{47}$ ) qui fait de l'œuvre un point de départ d'une extension de récits, de valeurs et d'actions plus large : il est ici question d'une pluralité de textes dans des langues différentes; de textes qui sont certes soudés par une relation d'interdépendance et qui se situent sur une ligne de temps précise - avec un avant et un après -, mais qui se croisent bien au-delà de tout «transfert » de A (œuvre originale, source) à B (œuvre traduite, cible). Bien au-delà de toute dimension exclusivement dérivative et de tout dialogue binaire, qui voit d'un côté la voix et la langue de l'auteur et de l'autre celles du traducteur. La fabrique de la Série Noire produit une pluralité de versions au sein de laquelle chaque texte est une œuvre distincte, soumise à différentes contraintes d'ordre littéraire et, plus généralement, culturel ; au sein de laquelle chaque texte est œuvre collective.

Dans ces conditions, s'intéresser comme le fait par exemple la critique génétique au processus de création, c'est-à-dire d'invention ${ }^{48}$, signifie avant tout s'affranchir de l'idée que l'invention est l'émanation d'une volonté auctoriale individuelle, que ce soit celle de l'auteur ou du traducteur, dont on essaierait de reconstruire et de restituer le mouvement; cela signifie, en somme, penser l'auctorialité d'abord comme fonction discursive et comme fonction collective: le vide, la "rupture ${ }^{49}$, laissé par la disparition de l'auteur et du traducteur, tant dans leur rôle d'origine que dans celui de principe de sens du texte, est occupé ici par l'entreprise éditoriale.

Observer les engrenages de la production des romans Série Noire à partir de ses archives permet de mettre en évidence un glissement majeur: la fonction-auteur remonte à l'équipe éditoriale tout entière; elle est assurée et exercée par l'éditeur et cela, comme l'a souligné Matthieu Letourneux, "indépendamment de tout acte d'énonciation $»^{50}$, car le jeu singularisant et souvent fictif des noms d'auteur et de traducteur ne se maintient que comme élément de la narration.

\section{NOTES}

1. Jean-Marc Gouanvic, Hard-boiled fiction et Série Noire, Paris, Classiques Garnier, 2018, p. 13, 37-49. 
2. Benoît Tadié, "La Série Noire et le roman noir américain ", dans A. Cerisier et F. Lhomeau (dir.), C'est l'histoire de la Série Noire (1945-2015), Gallimard, 2015, p. 106.

3. Lucia Quaquarelli et Adrien Frenay, "Faire américain. Fabbricare testi e autori : tradurre", Mediazioni, 27, 2020.

4. Daniel Ferrer, Logiques du brouillon. Modèles pour une critique génétique, Paris, Seuil, 2011, p. 16.

5. Adrien Frenay et Lucia Quaquarelli, «Ce n'est pas tout à fait Série Noire! Quand la traduction produit du roman noir », dans L. Quaquarelli, D. Cailleux et C. Denti (dir.), Expériences de traduction. Penser la traduction à travers ses pratiques, Bruxelles, Peter Lang, 2020, pp. 71-89.

6. Fabienne Durand-Bogaert, «Les deux corps du texte », Genesis, 38, 2014, p. 13.

7. Daniel Ferrer, Logiques du brouillon, op. cit., p. 16.

8. Matthieu Letourneux, Fictions à la chaine. Littérature sérielle et culture médiatique, Paris, Seuil, 2017, p. 44.

9. Ibid., p. 31.

10. Dès 1949, Marcel Duhamel insiste sur le caractère internalisé de la production des romans. Dans un « Rapport sur la Série Noire » (1949), il écrit au sujet des romans Série Noire : "nous les écrivons à la maison».

11. Il dispose d'un budget de $10 \%$ du prix de chaque exemplaire vendu qui doit servir au paiement des droits et des rémunérations.

12. Any Bonneval, «Marcel Duhamel ", La Gazette des Lettres, 5 août 1950, cité dans Frank Lhomeau, « Onze entretiens avec Marcel Duhamel », Temps Noir, 19, 2016, p. 155.

13. "Vingt nières et trois souris changent les truands en romanciers noirs ", Paris-Presse, 4 février 1954, cité dans Frank Lhomeau, « Onze entretiens avec Marcel Duhamel », op. cit., p. 164.

14. Frank Lhomeau, «La Série Noire de Marcel Duhamel 1945-1977 », A. Cerisier et F. Lhomeau (dir.), C'est l'histoire de la Série Noire (1945-2015), op. cit., p. 73.

15. Marcel Duhamel, Introduction au Catalogue de la Série Noire, Paris, Gallimard, 1955.

16. Ibid.

17. Voir la postface de Marcel Duhamel à sa traduction d'un passage de Poison Ivy parue dans L'arbalète $(9,1944$, p. 82) : «car ce qui lui [Cheyney] manque d'authenticité, de vécu, me paraît largement compensé par un humour - ou plutôt une truculence - très personnel dans son usage du slang, et surtout cette violence que l'auteur distribue de la première à la dernière page à un rythme frénétique... ».

18. "We want cinematic pace and suspense, if possible, but above all, authenticity. ", Lettre à Hope Leresche du 3 juin 1957.

19. Lettre à M. Sourdis du 6 octobre 1964.

20. Lucia Quaquarelli et Adrien Frenay, « Faire américain. Fabbricare testi e autori : tradurre », op. cit.

21. L'année de la parution dans la collection du premier roman écrit en français sans pseudonyme américanisant : Yvan Dailly, J'ai bien l'honneur..., 91, 1951.

22. Frank Lhomeau, «Les premiers Français de la "Série Noire", 1ère partie (1948-1953) », Temps Noir, 13, 2010, p. 43.

23. En tête de Cet homme est dangereux de P. Cheyney (2, juillet 1948) nous lisons : «Le roman détective a subi depuis quelques années dans les pays anglo-saxons une évolution suffisamment marquée pour que l'on puisse maintenant parler d'un genre nouveau. L'intérêt cesse de se porter sur la solution de l'énigme; c'est pour la psychologie des personnages, pour l'intensité de l'action, pour le pittoresque du langage que, depuis Dashiell Hammett, les nouveaux maîtres anglais et américains du roman policier veulent nous passionner. »

24. Texte rédigé par Marcel Duhamel, imprimé sur le rabat de la jaquette des romans publiés par la collection à partir de 1948.

25. Soit $48 \%$ des romans traduits publiés dans la collection. 
26. Dans l'exemplaire de Mr Denning Drives North (E. P. Dutton, New York, 1951. L'assassin revient toujours, tr. J.-G. Marquet, Gallimard, Série noire, $\mathrm{n}^{\circ} 172$, 1953) moins d'une dizaine de pages ne présentent aucune marque.

27. L'équipe de la Série Noire effectue son travail sur des états des textes angloaméricains très divers que Marcel Duhamel cherche à conserver en insérant dans les exemplaires des «Prière de retourner ». Le roman de Frank Kane, Dead Weight (publié en 1951, comme la traduction française) est présent uniquement sous la forme d'un manuscrit. D'autres romans sont présents en hardback et en paperback. Dans ce cas, la plupart du temps, un seul des deux exemplaires est annoté. Ainsi les exemplaires vierges $\mathrm{du}$ fonds ne constituent pas des indices probants d'absence d'intervention éditoriale.

28. L'étude approfondie de J.-M. Gouanvic permet par exemple de constater que des exemplaires peu marqués ou vierges de toute annotation sont malgré tout parfois largement coupés. Voir « La préparation du texte source en vue de la traduction/adaptation. Le cas de Shear the Black Sheep (David Dodge, 1943) traduit par Henri Robillot (Série noire, 1950)», op. cit., pp. 65-79 et surtout "L'abrègement de The Long Goodbye (1953/1954) par Henri Robillot et Janine Hérisson », op. cit., pp. 181-219. C'est cependant selon l'auteur de l'étude une exception dans le traitement des romans de Chandler, généralement peu ou pas coupés.

29. C'est le cas du roman d'Alec Coppel, Mr Denning Drives North, op. cit.

30. Une partie de ces marques peut être interprétée comme étant le fait du traducteur lors de son travail, comme la division du texte en segments avec mention du jour de la semaine ou de la date. 31. La Brigade des tueurs, tr. C. Grégoire, 925, 1965. L'exemplaire du fonds présente de très nombreuses marques.

32. Un ange est cassé, tr. G. Sollacaro, 454, 1958.

33. Nous reprenons en partie la typologie établie par Clem Robyns, «The Normative Model of Twentieth Century Belles Infidèles: Detective Novels in French Translation », Target. International Journal of Translations Studies, 1, 1990, pp. 23-42.

34. Au pied du mur, tr. G. Horst et J.-G. Marquet, 159, 1953.

35. Trois tondus et un pelé, tr. H. Robillot, 51, 1950. Pour une analyse approfondie du traitement éditorial de Shear the Black Sheep, voir J.-M. Gouanvic, op. cit., pp. 65-79.

36. L'assassin revient toujours, tr. J.-G. Marquet, 172, 1953.

37. Alerte à la fraîche, tr. J. Hérisson, 1577, 1973.

38. Voué au blanc-bleu, tr. J. Hérisson, 209, 1954.

39. Coup de torchon, tr. J. Hérisson, 183, 1953.

40. J.-M. Gouanvic, op. cit., pp. 147 et 149.

41. Le document fait référence à la «Série Rouge » comme d'une possibilité, ce qui laisse à penser qu'il date de 1949 mais on y lit aussi la phrase suivante qui pourrait situer la rédaction un peu plus tard, courant 1950 : «En 1949, Claude me fait remarquer que... ». Ce « rapport » est soit une version améliorée des documents cités précédemment, qui seraient alors des versions préparatoires, soit une synthèse ultérieure plus aboutie.

42. La liste en elle-même mérite d'être considérée puisqu'elle mentionne trois traducteurs dont deux ne publient pas de traductions dans la collection. Seul Jacques Legris traduit le 41 ainsi que le 76 (en collaboration avec Marcel Duhamel).

43. Le rythme de publication à partir de 1948 passe à deux titres par mois, d'où la multiplication.

44. CNRTL : https://cnrtl.fr/definition/fabrique.

45. Yvan Dailly, J'ai bien l'honneur..., op. cit., 1951. 
46. Tiphaine Samoyault, Vulnérabilité de l'œuvre en traduction, in Genesis, 38, 2014, pp. 57-68.

47. Sur l'idée de la traduction comme pratique herméneutique voir, entre autres, les réflexions d'Umberto Eco dans Dire quasi la stessa cosa. Esperienze di traduzione, Bompiani, Milano, 2003.

48. Daniel Ferrer, Logiques du brouillon, op. cit., p. 30

49. Voir à cet égard Michel Foucault, Qu'est-ce qu'un auteur ? Bulletin de la Société Française de Philosophie, 3, 1969, pp. 73-104.

50. Matthieu Letourneux, Fictions à la chaîne, op. cit., p. 15.

\section{AUTEURS}

\section{ADRIEN FRENAY}

Université Paris Nanterre

LUCIA QUAQUARELLI

Université Paris Nanterre 